\title{
A NEW PERSPECTIVE ON NEW MATERIALS
}

I Johannes Jobst \& Sense Jan van der Molen - DOI: https://doi.org/10.1051/epn/2018404 I Leiden Institute of Physics - Leiden University - Niels Bohrweg 2, 2333 CA Leiden - The Netherlands I E-mail: jobst@physics.leidenuniv.nl - molen@physics.leidenuniv.nl

\section{We live in an age of nanomaterials in which new materials are discovered almost every day. Moreover, we are starting to engineer material properties at the nanoscale. Hence, we need new tools to investigate different materials routinely and on small length scales.}

he ages of human history are classified by the materials used for the most innovative technologies at that time. Although we made a great leap from the biface in the stone ages to computers and the internet in our digital, connected silicon age, it is still difficult to predict which material will define future societies. Consequently, many new materials are under investigation today for a wide range of applications of tomorrow. These materials are typically only available in small quantities or are relatively inhomogeneous on the sub-micron scale. This limits the use of many optical techniques as they lead to an averaged result at best. Instead, what one wants are tools that can find tiny areas of interest on a sample and take meaningful data from it. In the best case, even local variations can be resolved. Good lateral resolution is crucial for materials where the properties are intentionally tuned by local, nanoscopic modifications. A particularly pronounced example of these are the so-called Van der Waals materials, artificial stacks of atomically thin sheets of layered crystals such as graphite (conductor), hexagonal boron nitride (insulator) or transition metal dichalcogenides (semiconductor). Isolated atomic planes can be reassembled into designer heterostructures made layer by layer in a precisely chosen sequence. The local assembly of these layers and their orientation with respect to each other strongly modify the properties of the whole stack [1].

\section{Measuring the Band Structure}

One of the most important characteristics of a material is its band structure. It describes which momenta $k$ and energies $E$ are allowed for electrons within the material, which, in turn, determines most electronic and optical properties. The band structure is the analogy of atomic 


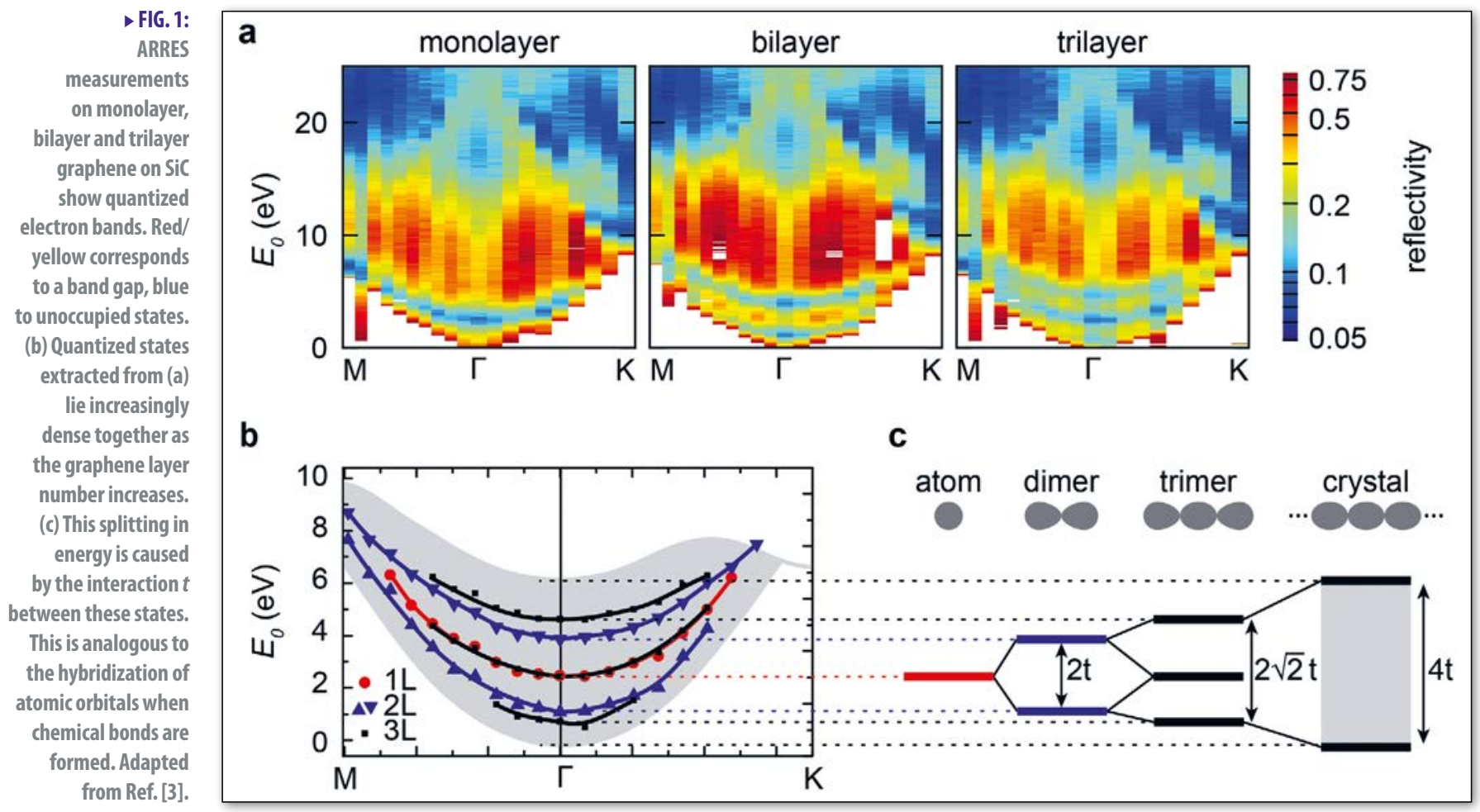

or molecular orbitals for crystals. Low-energy states are filled with electrons first while states at higher energy remain empty. This boundary between occupied and unoccupied electronic states is called the Fermi level. At even higher energies, the attractive interaction between electrons and atomic cores becomes so weak that electrons in states above this so-called vacuum level will leave the material.

Band structures have been a topic of intense research for almost a century providing some of the most important insights in condensed matter physics [2]. In particular, angle-resolved photoemission spectroscopy (ARPES) has become one of the standard characterization tools for any newly discovered material. In ARPES electrons are emitted upon illumination with photons due to the photo-electric effect. Due to momentum and energy conservation in this process, the recorded energy and momentum of these electrons is a direct representation of the occupied states of a material. The photo excitation cross-section is small, however, and therefore either large samples or very bright, focused light sources (e.g. synchrotrons) are needed.

In addition, many samples are non-uniform even below the length scale that can be resolved in ARPES ( $200 \mathrm{~nm}$ in state-of-the-art synchrotrons). This is particularly pronounced for Van der Waals materials. Consequently, we need another probe with higher interaction cross-section and shorter wavelength. Low-energy electron microscopy (LEEM, see Box 1) offers the perfect probe: low-energy electrons have sub-nanometer wavelength (above $2 \mathrm{eV}$ ) and their reflectivity is determined by unoccupied bands of the sample studied (see Box 2). Moreover, the energy $E_{0}$ and in-plane wave vector $k_{\|}$of the electrons used for imaging can be precisely chosen. Every pixel in the acquired images thus represent the local reflectivity 
as a function of $E_{0}$ and $k_{\|}$and consequently also the local unoccupied band structure. We baptized this new method angle-resolved reflected-electron spectroscopy (ARRES) [3] by analogy with the name of ARPES. In fact, ARRES and ARPES are complimentary techniques as the former probes unoccupied bands above the vacuum level, whereas the latter measures occupied bands below the Fermi level.

\section{From Quantized States to Extended Bands}

Due to this local character, we can use ARRES to study Van der Waals materials in the few-layer limit. We can, thus, study how to build up a band structure from the bottom up, layer by layer. Figure 1a shows ARRES measurements of the unoccupied band structure of monolayer, bilayer and trilayer graphene on silicon carbide. They all share a common region of high reflectivity (red area around $E_{0}=10 \mathrm{eV}$ ) that corresponds to a band gap. At lower energies, in contrast, the band structure of different layer number differs significantly. Monolayer graphene exhibits a pronounced band of unoccupied states (blue), while bilayer shows two and trilayer three of these bands. The single band in the monolayer system corresponds to a single interlayer state between the graphene layer and a carbon layer that terminates the $\mathrm{SiC}$ substrate (see Box 2). In bilayer graphene, a second interlayer state is present between the two graphene layers. Due to the close vicinity of the two states, they interact quantum mechanically. This leads to a splitting of the energy levels, analogous to the tight-binding description of molecules: when the orbitals of two atoms overlap, they hybridize into a lower 'binding' and a higher-energy 'anti-binding' molecular orbital. In this analogy the individual interlayer states in graphene play the role of atomic orbitals. The important difference is that these 'atomic' states are quantized only in the out-of-plane direction, but quasi-infinite within the plane. They can thus be considered 'two-dimensional atoms. Consequently, the splitting of the bands observed in ARRES (Fig. 1a) is a direct measure for the interaction strength of interlayer states in graphene.

Moreover, if we keep adding layers to the system, we can observe the evolution of individual quantum objects into the continuum of an extended crystal. Every additional layer contributes another interlayer state that interacts with the other states. This means that their spacing becomes smaller and smaller and eventually they all overlap to form a continuous electronic band (Fig. 1b). This case can be observed in graphite, which is an almost-infinite stack of graphene layers. Figure 2a shows the ARRES measurement of the unoccupied graphite bands (top panel) together with ARPES measurements of the occupied part (lower panel) acquired in the same instrument. Theoretical calculations (black lines) correspond very well with the measured data and, indeed, a continuous electronic band is observed between 0 and $\sim 10 \mathrm{eV}$ (yellow in Fig. 2a).

The wealth of information that is encoded in the unoccupied band structure of a material can be witnessed from the comparison of ARRES measurements on graphite and hexagonal boron nitride (hBN) in Fig. 2a and b. Although

\section{BOX 2: SELECTING THE ELECTRON MOMENTUM}

To measure any part of the band structure, the presence of electronic states has to be obtained as a function of electron energy $E_{0}$ and in-plane momentum $k_{\| 1}$. In LEEM, we can easily achieve this by tilting the incoming electron beam using electrostatic deflectors (like in an old TV based on cathode ray tubes) as sketched in (a). In fact, we can tilt the beam such that $k_{\| 1}$ follows the high-symmetry lines connecting the Gamma ( $\Gamma), \mathrm{K}$ and $\mathrm{M}$ points (b). They reflect the hexagonal symmetry of most Van der Waals materials and measuring the bands along those lines captures most of the physical properties. Normal incidence, i.e., $k_{\|}=0$ (left in a), corresponds to the Gamma point.

a

w-energy

electrons

graphene

SiC b

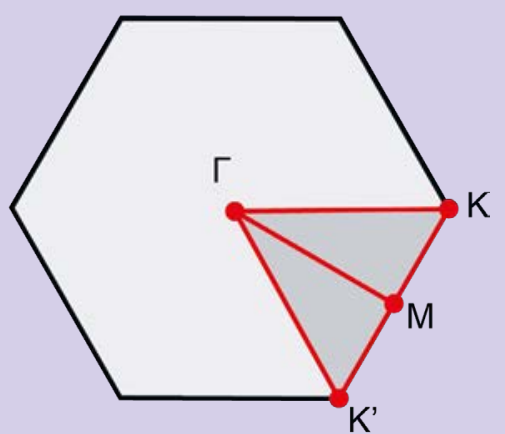




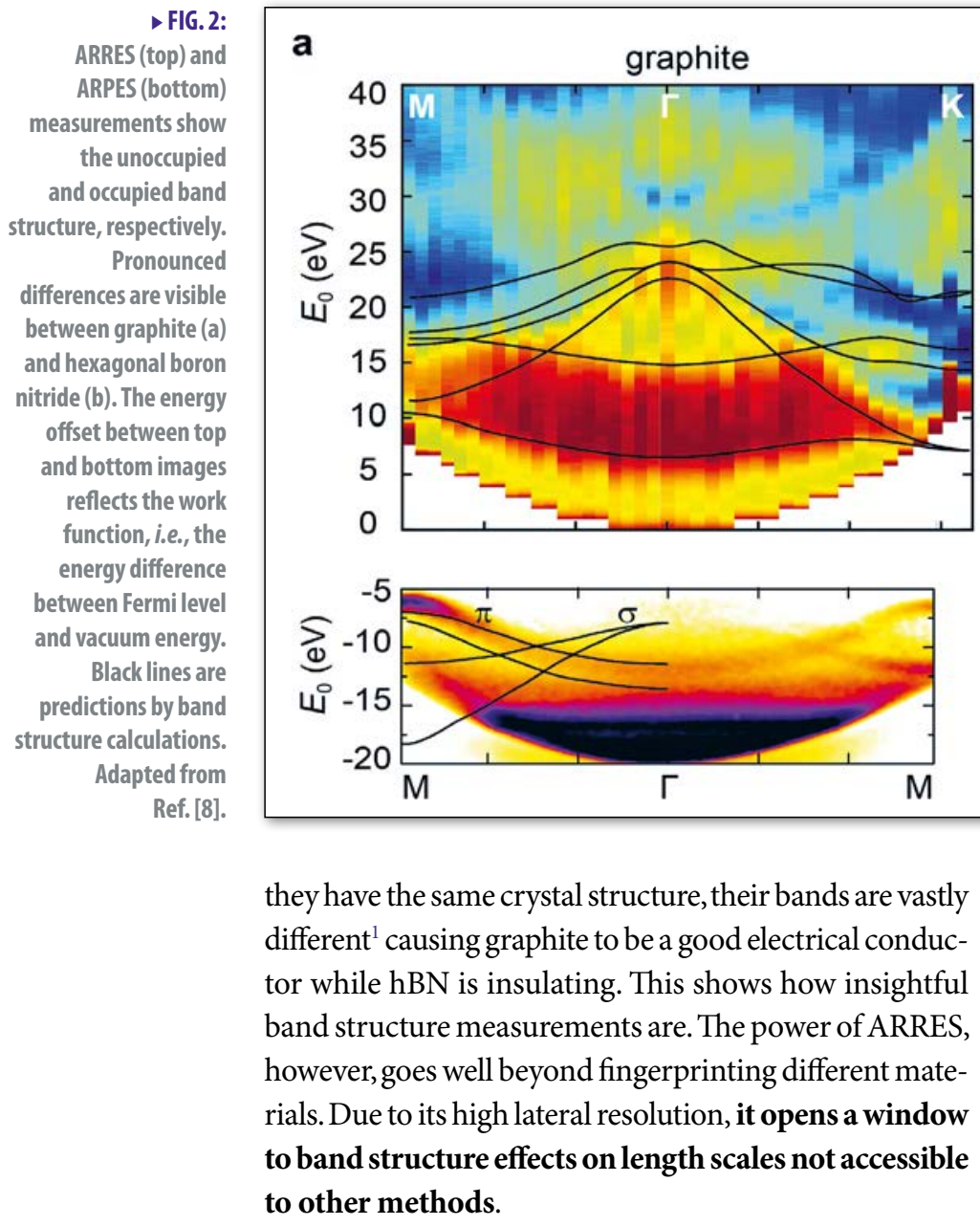

\section{Band Structure Engineering}

This capability is crucial for the investigation of samples where the band structure is tailored by local modifications. In Van der Waals materials such band structure engineering can be achieved by the assembly of individual two-dimensional layers from different host crystals. This Lego-like approach renders it possible to construct materials from scratch that do not exist naturally. The large variety of available $2 \mathrm{D}$ crystals means that a sheer endless number of combinations are possible. Those Van der Waals materials can be further tailored by changing the stacking order of layers, the strain within layers or the rotation angle with which they are placed on top of one another $[9,10]$. In addition, the layers and interfaces can be decorated with atoms or molecules. All those tuning parameters lead to local variations in the band structure that determine the final properties of the Van der Waals material. ARRES yields new insights into the band structure on those length scales [8]. A better fundamental understanding of this band structure engineering may allow us to design novel materials with desired properties for future technologies.

\footnotetext{
${ }^{1}$ The title image is a constant-energy cut through the hBN band structure at $12 \mathrm{eV}$ obtained in ARRES.
}
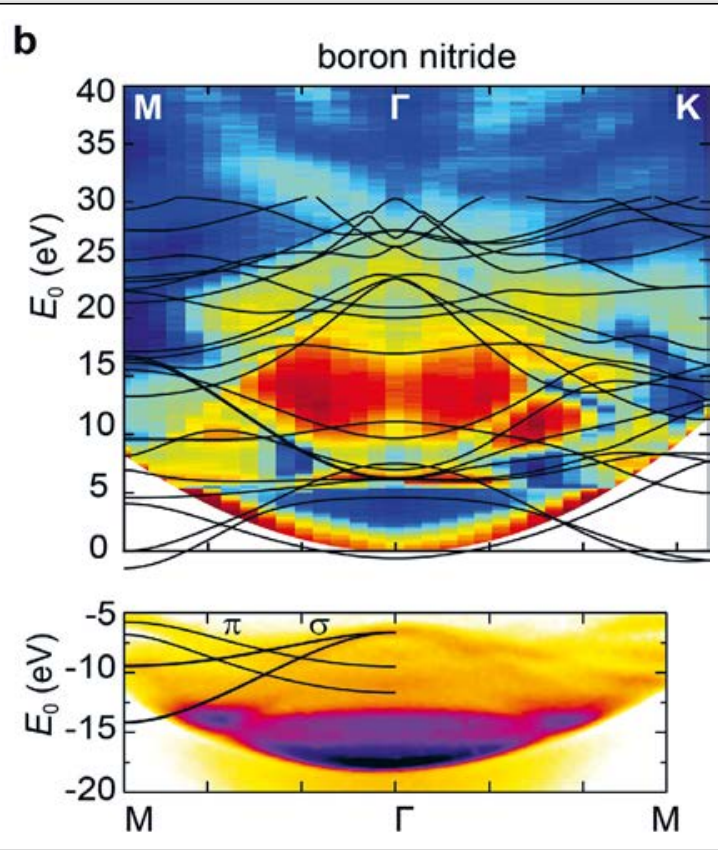

\section{About the Authors}

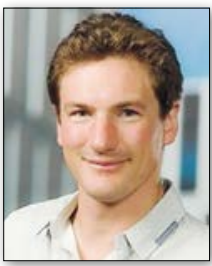

Johannes Jobst is postdoc at Leiden University and Columbia University, New York. He received his $\mathrm{PhD}$ in the group of Heiko Weber at the University of Erlangen-Nuremberg for work on quantum transport in graphene. Johannes won a prestigious, personal VENI grant in 2015 and was one of three KNAW delegates to the Lindau Nobel Laureates Meeting in 2016.

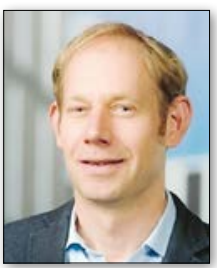

Sense Jan van der Molen is a full professor at Leiden University. After obtaining his $\mathrm{PhD}$ at the Vrije Universiteit in Amsterdam, he switched to the field of molecular charge transport, working in Groningen with Bart van Wees and Ben Feringa, as well as in Basel. Since 2007, he is leading a group in Leiden.

\section{References}

[1] A. K. Geim, I. V Grigorieva, Nature. 499, 419 (2013).

[2] A. Damascelli, Rev. Mod. Phys. 75, 473 (2003).

[3] J. Jobst, J. Kautz, D. Geelen, R. M. Tromp, S. J. van der Molen, Nat. Commun. 6, 8926 (2015).

[4] R. M. Tromp, Ultramicroscopy. 111, 273 (2011).

[5] R. M. Tromp, J. B. Hannon, W. Wan, A. Berghaus, O. Schaff, Ultramicroscopy. 127, 25 (2013)

[6] E. Bauer, Surface Microscopy with Low Energy Electrons (Springer New York, New York, NY, 2014; http://link.springer. com/10.1007/978-1-4939-0935-3).

[7] J. I. Flege, E. E. Krasovskii, Rapid Res. Lett. 8, 463 (2014).

[8] J. Jobst et al., Nat. Commun. 7, 13621 (2016).

[9] Y. Cao et al., Nature. 556, 80 (2018).

[10] Y. Cao et al., Nature. 556, 43 (2018). 